\title{
Guest editorial: Scientific and practical issues associated with piglet castration
}

\author{
S. A. Edwards ${ }^{1}$, E. von Borell ${ }^{2}$ and M. Bonneau ${ }^{3}$
}

${ }^{1}$ Newcastle University, Newcastle NE1 7RU, UK; ${ }^{2}$ Martin-Luther-University of Halle-Wittenberg, Halle, Germany; ${ }^{3}$ INRA, UMR 1079, F-35042 Rennes, France

In current livestock farming practice, a number of painful procedures, often termed as mutilations, are carried out on animals for purposes associated with the prevention of injurious behaviour, identification of individuals, improving ease of management, or enhancement of product quality. Amongst these, the most widespread is the practice of castration of male animals, done in most species to avoid unwanted breeding, prevent the development of undesirable and possibly injurious male sexual behaviours, and prevent the development of unpalatable male odours and flavours in the meat. Whilst the practice of castration is subject to some legal restriction, it is still the case that each year many millions of farm animals are subject to a painful procedure that is often carried out without anaesthesia or analgesia. This has become a subject of increasing public concern, which has been particularly focussed on pigs because the anatomy of this species has meant that only surgical methods have been possible until very recently. As a result of this concern, the European Union (EU) commissioned a specific scientific review of the welfare issues associated with the castration of piglets by European Food Safety Authority (EFSA) in 2004, and subsequently a Specific Support Action (Attitudes, practices and state of the art regarding piglet castration in Europe, PIGCAS) to review the state of the art and critically appraise possible alternatives to the current widespread practice of surgical castration without pain relief.

This themed volume of Animal contains a series of papers arising from the PIGCAS project. At the outset of the project, it was apparent that there was limited information on the current practices for pig castration in different EU member states. This was addressed by carrying out an EU-wide survey, whose results are reported in the first paper (Fredriksen et al., 2009). This shows wide variation in the timing, methods and extent of pain relief used in practice. Whilst EFSA has previously reviewed the welfare aspects of piglet castration (EFSA, 2004; Prunier et al., 2006), there has since been substantial scientific activity in this subject area and more extensive evaluations of some of the existing and newly available practical alternatives. An updated review is therefore the subject of the second paper (von Borell et al., 2009). The relative merits of the possible solutions involving different approaches to anaesthesia and analgesia have generated much scientific debate and there is still not a consensus on this subject. This is shown by the letters to the editor which are also published in this issue (Gerritzen et al., 2009; Wright et al., 2009), and which relate to comment on an earlier paper on the subject published in Animal (Gerritzen et al., 2008).

Castration of male pigs may offer some advantages for subsequent ease of management and reduction in undesirable male sexual behaviours that may pose a risk to welfare of other animals. However, the primary reason for the continuation of castration as a widespread practice is the need to safeguard meat quality. This aspect is reviewed in the third paper (Lundström et al., 2009), which gives particular attention to the risk of boar taint in the carcass and the potential for on-line methods for its detection. A further paper (Zamaratskaia and Squires, 2009) reviews the biochemical regulation of boar taint compounds in more detail and explores possible future immunological, nutritional and genetic approaches to minimise the problem. Ultimately, in the assessment of different future strategies to enhance animal welfare whilst safeguarding meat quality, it is important that the scientific knowledge is integrated with a review of the economic implications. This is the subject of the final paper in the series (de Roest et al., 2009). Together, these five review papers provide a comprehensive and current analysis of the issue of castration in pigs, which can inform both future scientific initiatives and policy decisions in this important subject area.

\section{References}

Fredriksen B, Font i Furnols M, Lundström K, Migdal W, Prunier A, Tuyttens F and Bonneau M 2009. Practice on castration of piglets in Europe. Animal 3 , 1480-1487.

de Roest K, Montanari C, Fowler T and Baltussen W 2009. Resource efficiency and economic implications of alternatives to surgical castration without anaesthesia. Animal 3, 1522-1531.

EFSA 2004. Welfare aspects of the castration of piglets. Scientific Report of the Scientific Panel for Animal Health and Welfare on a request from the Commission related to Welfare Aspects of the Castration of Piglets, European Food Safety Authority AHAW/04-087.

Gerritzen MA, Kluivers-Poodt M, Reimert HGM, Hindle V and Lambooij E 2008. Castration of piglets under $\mathrm{CO}_{2}$-gas anaesthesia. Animal 2 1666-1673. 
Gerritzen M, Kluivers-Poodt M, Reimert H, Hindle V and Lambooij B 2009. Response to the Letter to the Editor on the surgical castration of piglets. Animal 3, 1476-1477. Lundström K, Matthews KR and Haugen J-E 2009. Pig meat quality from entire males. Animal 3, 1497-1507.

Prunier A, Bonneau M, von Borell EH, Cinotti S, Gunn M, Fredriksen B, Giersing $M$, Morton DB, Tuyttens FAM and Velarde A 2006. A review of the welfare consequences of surgical castration in piglets and the evaluation of nonsurgical methods. Animal Welfare 15, 277-289. von Borell E, Baumgartner J, Giersing $M$, Jäggin N, Prunier A, Tuyttens FAM and Edwards SA 2009. Animal welfare implications of surgical castration and its alternatives in pigs. Animal 3, 1488-1496.

Wright AJ, Whiting M and Taylor A 2009. Letter to the Editor on the surgical castration of piglets. Animal 3, 1474-1475.

Zamaratskaia G and Squires EJ 2009. Biochemical, nutritional and genetic effects on boar taint in entire male pigs. Animal 3, 1508-1521. 